VALUE-BASED OPHTHALMOLOGY

\title{
Cost utility of screening and treatment for early age related macular degeneration with zinc and antioxidants
}

\author{
C Hopley, G Salkeld, J J Wang, P Mitchell
}

Series editors: Melissa

Brown and Gary Brown

See end of article for authors' affiliations .....................

Correspondence to: Professor Paul Mitchell, University of Sydney Department of

Ophthalmology,

Westmead Hospital,

Hawkesbury Road,

Westmead, NSW 2145

Australia;

paul_mitchell@

wmi.usyd.edu.au

Accepted for publication 25 October 2003
Br J Ophthalmol 2004;88:450-454. doi: 10.1136/bjo.2003.035279

Aim: To assess the cost effectiveness of high dose zinc and antioxidants for delaying and reducing the progression of early age related macular degeneration (AMD).

Background: $A M D$ is the leading cause of severe vision impairment and blindness in older people throughout the developed world. It currently affects around 420000 people in the United Kingdom.

Methods: A cost utility analysis (CUA) was conducted to estimate the cost per quality adjusted life year (QALY) for screening a cohort of men and women, aged 55 years and over, for early AMD and then treating them with zinc and antioxidants. The incremental CUA was based on a decision analytic model, comparing screening with a no screening comparator (current practice). Extensive one way sensitivity analysis of parameters was conducted to determine the robustness of the model.

Results: In this model the cost effectiveness of screening for early AMD was $£ 22722$ per quality adjusted life year (QALY) saved. The cost per QALY decreased to $£ 18948$ if photodynamic therapy with verteporfin savings were included.

Conclusions: Screening for, and prophylactic treatment of, early AMD is estimated to cost around $£ 22700$ per QALY saved. This cost falls within accepted levels to warrant further investigation. These findings have implications for ophthalmic practice and healthcare planning.
1 ndividuals with vision impairment experience reduced quality of life, difficulties with activities of daily living, and may be socially isolated. ${ }^{1}$ Age related macular degeneration (AMD) is the leading cause of severe visual impairment for older people in the developed world..$^{2}$ In the United Kingdom the disease affects around 420000 people and an estimated 214000 people have registrable vision impairment secondary to AMD. ${ }^{3}$ Those with visual impairment or blindness use considerable healthcare resources. ${ }^{45}$ They also require community support services to maintain independent living in the community. ${ }^{6}$ With an ageing population the burden of disease from AMD is expected to increase over time.

There are limited options for the treatment of AMD. Laser is effective, but only in around $15 \%$ of neovascular AMD cases. ${ }^{7}$ Photodynamic therapy (PDT) with verteporfin is applicable in a higher percentage of situations ${ }^{8}$ but is expensive at around $£ 4800$ per patient." The National Institute for Clinical Excellence (NICE) is examining its cost effectiveness and it is not currently available on the National Health Service.

Smoking is the most important modifiable risk factor for AMD.$^{10}$ Current smokers develop AMD three to four times more frequently and 10 years earlier than non-smokers. ${ }^{11}{ }^{12}$ However, the ongoing reductions in the prevalence of smoking in developed countries ${ }^{13-15}$ will not be anywhere near large enough to dramatically alter the incidence of AMD.

Research has been conducted into ways of preventing the progression of early AMD. The US National Eye Institute funded the Age Related Eye Disease Study (AREDS), a double masked prospective clinical trial, examined the potential benefit of a prophylactic high dose zinc and antioxidants formulation in early AMD, compared with placebo. ${ }^{16}$ The prophylactic formulation significantly retarded or prevented the development of reduced visual acuity and neovascular or atrophic AMD, in persons with early AMD (AREDS categories 3 and 4 , table 1), compared with placebo. There were no significant side effects attributable to treatment and compliance levels were good.

These finding are of significance as they provide the first hard evidence that AMD progression may be prevented through a screening and early treatment approach. ${ }^{17}$ The question remains, however, as to whether it makes economic sense to screen and treat an older population with early AMD.

The aim of this study was to model the cost per quality life year gained (QALY) of population based screening and treatment of early AMD with high dose zinc and antioxidants, compared with no screening or treatment. In doing so our aim was to quantify existing knowledge about the screening strategy-with its attendant uncertainty-in a decision analytical model. This could provide insight into the value of collecting new information for specific parameters, like preference based measures of visual acuity related quality of life, and to refine cost effectiveness estimates for the purpose of funding decisions. The study was conducted from a third party payer perspective.

\section{METHODS}

\section{Model overview}

A multicohort, combined prevalence and incidence decision analytic model was created to synthesise data over a total of four ( 5 year) screening cycles. Screening was modelled for men and women in four overlapping groups, using the following cut-off ages: $\geqslant 55$ years, $\geqslant 65$ years, $\geqslant 70$ years, and $\geqslant 75$ years. The model assumed optician based screening (performing dilated funduscopic examination) to identify the number of people with early AMD, who were then treated with the prophylactic high dose zinc and antioxidant formulation. Outcomes were modelled using data from the two AREDS end points; (1) incremental visual acuity events

Abbreviations: $A M D$, age related macular degeneration; CUA, cost utility analysis; GA, geographic atrophy; QALY, quality adjusted life year; RPE, retinal pigment epithelium 
Table 1 Age Related Eye Disease Study (AREDS) definitions and details

\section{AREDS categories}

All categories had a best visual acuity of at least $6 / 9$ (the study eye) at baseline.

Category 1: Range from completely normal macula to a few small drusen.

Category 2: Multiple small drusen, single or non-extensive intermediate size drusen, pigment abnormalities.

Category 3: Absence of advanced age related macular degeneration (AMD) in both eyes, with at least one large drusen at macular centre, or extensive intermediate drusen or geographic atrophy (GA) not involving the central macula.

Category 4: No advanced AMD in one eye, with vision impairment from AMD in fellow eye

Definitions of AREDS outcomes

Neovascular events: Progression to advanced AMD for a study eye was defined by:

(1) laser photocoagulation or other treatment for choroidal

neovascularisation, or

(2) any of the following: GA involving the macula centre, non-drusenoid retinal pigment epithelial (RPE) detachment, serous or haemorrhagic

retinal detachment, haemorrhage under the retina or RPE, and/or subretinal fibrosis.

Visual acuity events: A decrease in best corrected visual acuity score from baseline, of 15 or more letters in a study eye (a doubling or more of the visual angle, from baseline equivalent $6 / 9$ or better, to $6 / 18$ or worse).

Incremental output: the difference between treatment and placebo outcomes.

Study population characteristics

(1) AREDS: $96 \%$ European origin, age range $55-80$ years, median age 69 years. Study population garnered from medical records, patient lists, screening at public gatherings, other. Size 4757.

(2). Blue Mountains Eye Study: 98\% European origin, age range 4997 years, median age 66 years. Population based study. Size 3654 . AREDS zinc and antioxidant formulation: Zinc $80 \mathrm{mg}$ (+ copper $2 \mathrm{mg}$ ), vitamin C $500 \mathrm{mg}$, vitamin E $400 \mathrm{IU}, \beta$ carotene $15 \mathrm{mg}$.

Compliance levels in AREDS study: around $75 \%$ of tablets taken by $71 \%$ of patients.

Adverse events: Increased frequency of yellow skin reported by taking antioxidants.

No differences noted between groups for other potential adverse events.

were used to calculate the QALYs associated with the treatment arm, and (2) incremental neovascular events were used to calculate incremental cost savings from the reduced number of laser and PDT treatments (table 2). The annual costs associated with screening and prophylaxis accrued to the treatment arm alone. Costs were converted into pounds through a purchasing power parity calculation. Both QALYs and costs were discounted at a rate of $6 \%$.

Table 2 Methodology used in calculating model outputs using Age Related Eye Disease Study (AREDS) and other data

\section{Simplified model calculations:}

(1) Multiplying: (census data) $\times$ (incidence/prevalence) $\times$ (life expectancy). This gives the annual surviving prevalence and incidence numbers. These numbers were then multiplied by:

(Incremental AREDS visual acuity events figure) $\times$ (calculated utility figure). This gives annual incremental utilities, enabling quality adjusted life years (QALYs) to be calculated.

(Incremental AREDS neovascular events figure) $\times(\%$ of lesions applicable for treatment estimate $) \times(\%$ actually treated estimate $) \times($ unit cost $)$. This gives the annual incremental laser or PDT cost saving associated with the reduced use of the relevant therapy.

(2) The total number of people screened by optometrists was calculated from census and unit cost data and accrued to the treatment arm alone. (3) The cost of ongoing antioxidant and zinc treatment for all AREDS category 3 and 4 was calculated on an annual basis from screened survivors and accrued to the treatment arm alone. (4) Final cost utility output = relevant cost/QALY.

\section{Epidemiology data}

The Blue Mountains Eye Study database provided estimates of incidence and prevalence associated with AREDS categories 3 and 4, as well as visual impairment data. ${ }^{18}$ The data used were assumed to be representative of the Australian population of similar age and sex.

\section{Age Related Eye Disease Study (AREDS) data}

The differences between placebo and treatment groups were significant for both the visual acuity (OR 0.76; $99 \%$ CI 0.54 to 0.99 ) and neovascular (OR 0.62 ; $99 \%$ CI 0.43 to 0.90 ) end points. ${ }^{16}$ The visual acuity end points were based on the patient either losing vision in the better seeing eye or losing vision in both eyes simultaneously when vision had initially been good. The first 7 years of AREDS data were extrapolated, using trend lines, to produce likely future rates of progression. To obtain trend lines with the highest $R^{2}$ values (explaining most of the modelled variation), we used a polynomial approach in one and a linear approach in the other.

\section{Cost identification data}

A literature search was conducted in order to identify costs, supplemented by expert opinion through the authors' empirical experience of AMD resource use. The study included only relevant variable incremental costs (table 3). Costs were measured according to yearly use per affected person. Schedule fees were obtained from published 2001 Australian Medicare benefits schedule data (www.health. gov.au/pubs/mbs/mbs6/itemcode.htm). High dose zinc and antioxidants formulations were costed at market rates. Repeat population screening was modelled to occur every 5 years, with all costs accruing to the first year without any amortisation. Other costs such as capital expenditure, overheads costs, and certain labour costs were assumed to be equivalent in both the treatment and placebo arms and were not examined. Indirect costs were not assessed. No costs associated with the implementation or ongoing running of such a screening programme were included in the study.

\section{Other data sources and assumptions}

The Australian Bureau of Statistics provided census based population projection data ${ }^{19}$ and Australian Life tables. ${ }^{20}$ Expert opinion (PM) regarding the percentage of neovascular lesions applicable for, and the percentage of those actually receiving, laser and PDT therapy, was obtained before running the model. Laser and PDT costs were based on two and four treatments per person per year respectively. Australian federal government funding for PDT was assumed to be indefinitely extended.

\section{Utility assessment}

Preference based measures of vision related quality of life (utilities) were extracted from a paper by Sharma et al. ${ }^{21}$ The incremental utility values were derived using the following patient based utility formula:

$$
\text { Utility }=(0.374)(\text { best visual acuity })+0.514 .{ }^{21}
$$

The incremental visual acuity events associated with the placebo arm enables an annual calculation of utilities, and hence QALYs, that accrue with prophylactic treatment. The calculation results in a reduction in utility of 0.117 with every predicted visual acuity event.

\section{Purchasing power parity}

Purchasing power parity (PPP) rates were used to convert costs between currencies. Under PPP, exchange rates are adjusted to eliminate the differences in price levels between 
Table 3 The unit cost breakdown by category with Medicare benefits schedule (MBS) item numbers where relevant

\begin{tabular}{llll}
\hline Category & Source & AS & $£$ \\
\hline Photodynamic therapy & & & \\
Initial consultation & MBS 104 & 67 & 32 \\
Fluorescein angiogram & MBS 11212/15 & 162 & 78 \\
Verteporfin 15 mg vial & Federal health budget & 2100 & 1014 \\
Photodynamic therapy & MBS 42884 & 360 & 174 \\
Other consultations & MBS 108 & 33 & 16 \\
Total for 4 treatments & & 10653 & 5144 \\
Laser & MBS 104 & 67 & 32 \\
Initial consultation & MBS 11212/15 & 162 & 78 \\
Fluorescein angiogram & MBS 42809 & 351 & 170 \\
Fluorescein angiogram & MBS 108 & 1226 & 16 \\
Other consultations & & 54 & 592 \\
Total for 2 treatments & MBS 10900 & $\mathbf{2 1}$ & $\mathbf{2 6}$ \\
Screening per patient & Blackmores Ltd & & \\
Zinc and antioxidants & & & \\
\hline
\end{tabular}

countries and better reflect the real value of money when converting the costs of goods and services from one currency to another (www.pacific.commerce.ubc.ca/xr/PPP.html). Organisation for Economic Cooperation and Development (OECD) rates were used (www.oecd.org/std/ppp) to derive the 0.483 factor used to convert Australian dollars to pounds sterling.

\section{Discounting}

A discount rate is necessary to reflect the preference society has for delaying costs to a future date and enjoying benefits now. A real discount rate of $6 \%$ (UK Treasury rate) was used for both costs and QALYs, as recommended by Drummond et al. ${ }^{22}$

\section{Sensitivity analysis}

A preliminary analysis of the model, using a percentage change in input values, was conducted for all relevant uncertain variables. Those most affecting the incremental cost effectiveness ratios (cost per QALY) were selected for further evaluation in a one way sensitivity analysis. A range of values reflecting the inherent uncertainty for each selected variable were tested, including a QALY discount rate of $0 \%$ as suggested by Drummond et al. ${ }^{22}$

\section{RESULTS}

\section{Cost utility output}

In the 65 years and over age group, the cost per QALY was $£ 22722$ (table 4). This decreased to $£ 18948$ when the savings accruing owing to reduced numbers of PDT treatments were included. In the 70 years and over age group the equivalent cost per QALY was $£ 22$ 937. This decreased to $£ 19087$ when the savings accruing as a result of reduced numbers of PDT treatments were included.

\section{Marginal cost effectiveness ratio data}

The marginal cost effectiveness of lowering the commencement age of extending screening and treatment for AMD from 70 years to 65 years was $£ 20$ 849. This decreased to $£ 17526$ when the savings accruing to reduced numbers of PDT treatments were included.

\section{Results of sensitivity analysis}

The initial analysis, using a fixed percentage change in input values, showed the model to be most sensitive to, in decreasing order, monthly treatment costs (zinc and antioxidants), incremental utility value, QALY discount rate, real discount rate, and screening costs. Likely variability or suggested fixed values were then tested in the one way sensitivity analysis (fig 1). This resulted in a range of incremental cost effectiveness ratios from £1l 299 to $£ 28$ 282. The lowest value was obtained when the QALY discount rate was reduced to $0 \%$ and the highest when incremental utilities were reduced to 0.94 . Overall, the sensitivity analysis confirmed the model to be robust.

\section{DISCUSSION}

To our knowledge this is the first study of its kind and therefore we were unable to compare our estimates with those from other models. We endeavoured to follow the ISPOR guidelines for good practice in modelling. ${ }^{23}$ The model was based on data from the AREDS ${ }^{16}$ and the Blue Mountains Eye Study, ${ }^{24}$ which have both been extensively peer reviewed. Their study population characteristics are similar (table 1) and we believe the core model outputs are generalisable to similar populations groups-namely older people of European origin. The unit cost data were taken from an Australian setting and converted to pounds through a purchasing power parity calculation to better reflect likely

\begin{tabular}{|c|c|c|c|c|}
\hline \multirow[b]{3}{*}{ Screening age } & \multirow{2}{*}{\multicolumn{2}{|c|}{$\begin{array}{l}\text { Excluding PDT savings } \\
\text { Cost per QALY }\end{array}$}} & \multirow{2}{*}{\multicolumn{2}{|c|}{$\begin{array}{l}\text { Including PDT savings } \\
\text { Cost per QALY }\end{array}$}} \\
\hline & & & & \\
\hline & $\bar{£}$ & AS & $\bar{£}$ & AS \\
\hline \multirow{4}{*}{$\begin{array}{l}55+ \\
65+ \\
70+ \\
75+\end{array}$} & 23704 & 49092 & 19963 & 41344 \\
\hline & 22722 & 47058 & 18948 & 39242 \\
\hline & 22937 & 47502 & 19087 & 39528 \\
\hline & 23422 & 48506 & 20030 & 41482 \\
\hline
\end{tabular}




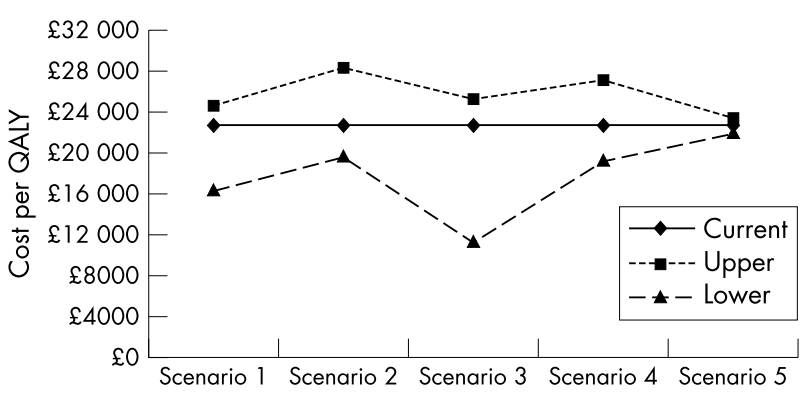

\begin{tabular}{lcrrr}
\hline & Variable & Lower & Current & Upper \\
\hline Scenario 1 & Monthly treatment cost & $£ 6.76$ & $£ 10.14$ & $£ 11.11$ \\
Scenario 2 & Incremental utility & 0.135 & 0.117 & 0.094 \\
Scenario 3 & QALY discount rate & $0 \%$ & $6 \%$ & $7 \%$ \\
Scenario 4 & Real discount rate & $8 \%$ & $6 \%$ & $4 \%$ \\
Scenario 5 & Screening cost per patient & $£ 21.73$ & $£ 26.17$ & $£ 31.39$ \\
\hline
\end{tabular}

Figure 1 One way sensitivity analysis of incremental cost effectiveness ratios (cost per quality adjusted life year or QALY) for each scenario.

UK costs. The sensitivity analysis gives a range of effects that enable robust cost generalisation across similar population groups.

The measurement of utilities originated to deal with rational decision making when confronted with uncertainty. ${ }^{22}$ Utilities, by convention, vary from 1.0 (perfect health) to 0.0 (death). Brown et al have shown that the utilities associated with ophthalmic disease are most highly correlated with visual acuity in the better seeing eye $\mathrm{e}^{25-29}$ and exhibit good retest reliability. ${ }^{30}$ The pathological cause of the visual impairment is of lesser importance, compared with visual acuity, as far as utility values are concerned. ${ }^{29}$ The effect on an individual of a "visual acuity event," reducing better eye visual acuity from $6 / 9$ to $6 / 18$ or worse, would be significant. Most noticeably, it would result in the person losing their licence to drive and it would make many activities of daily life more difficult to perform. Hence, the authors believe the calculated 0.117 incremental utility value for visual impairment is not unrealistic. Other valuations of the change in utility associated with a similar change in visual acuity, are of this order. ${ }^{27}$

Screening was modelled to occur every 5 years. Recent research indicates that diabetic retinopathy screening may be adequately performed every 3-5 years, rather than yearly as previously recommended. ${ }^{31}$ Similarly, the authors felt that yearly screening would be too onerous considering the numbers, associated costs, and diminishing potential for benefit.

We believe we have been conservative in our approach to costing. Many, if not most, older people already attend their optometrist or ophthalmologist on a regular basis and would not require a separate "screening" visit. We did not attempt to diminish screening costs by taking this into account. An optician based AMD screening programme would by default also screen for diabetic retinopathy, glaucoma, and other eye conditions. The non-AMD benefits accruing from this screening effort have also not been modelled. The cost utility output is most sensitive to the monthly cost of the high dose zinc and antioxidant formulation. Yet this price would almost certainly fall in the event of the screening programme coming to fruition, owing to increased economies of scale in production. All of these factors would potentially lead to a meaningful reduction in the actual cost per QALY.
Targeted screening, using risk factors, should arguably form part of routine optometric and ophthalmic practice. People who should be screened include those with a family history (affected sibling or parent), smokers, and those with an only eye. People with AMD in one eye should already routinely be prescribed the treatment, unless contraindicated. Training issues arise from the AREDS findings. Opticians, optometrists, and ophthalmologists need to be educated into the findings of the study, the categories of early AMD likely to benefit from treatment and the potential for targeted screening.

We made use of expert opinion estimations (PM) in the modelling process and AREDS categories 3 and 4 incidence data had to be estimated from the available data. Ideally peer reviewed data would be available for the modelling process.

\section{CONCLUSIONS}

AMD is the major cause of severe vision impairment in older, westernised, populations and has a severe impact on the quality of life. Treatments are available, but are often inapplicable for the disease state and can be very expensive. The rates of progression of early to late AMD can be reduced by high dose supplements, but require screening. Targeted screening should arguably be part of routine optometric and ophthalmic practice. Our decision analysis model demonstrated a cost of around £22 800 per QALY gained and suggest the $\geqslant 65$ years group to be the most efficient one to screen of the four assessed. Although there are no absolute standards for cost effectiveness, Canadian and now, de facto, American guidelines proposed by Laupacis et a ${ }^{32}$ put this into the moderate cost effectiveness category. This tends to indicate that an early AMD screening and treatment programme should be further assessed for possible implementation. We hope to have laid the foundations for future work and to have enabled the identification of areas of uncertainty, which can be further studied.

\section{ACKNOWLEDGEMENTS}

We thank Dr Rick Ferris III for kindly providing the neovascular events data from AREDS database.

\section{Authors' affiliations}

C Hopley, J J Wang, P Mitchell, Centre for Vision Research, Department of Ophthalmology and the Westmead Millennium Institute, University of Sydney, Australia

G Salkeld, School of Public Health, University of Sydney, Australia

This study was supported by an Initiating Grant from the Westmead Millennium and Save Sight Institutes, University of Sydney.

\section{REFERENCES}

1 Bishop BMP. The national strategy for an ageing australia. healthy ageing, Discussion Paper. 1-24. Canberra: Commonwealth of Australia, 1999.

2 Wang JJ, Foran S, Mitchell P. Age-specific prevalence and causes of bilateral and unilateral visual impairment in older Australians: the Blue Mountains Eye Study. Clin Exp Ophthalmol 2000;28:268-73.

3 Owen CG, Fletcher AE, Donoghue $M$, et al. How big is the burden of visual loss caused by age related macular degeneration in the United Kingdom? $\mathrm{Br} J$ Ophthalmol 2003;87:312-7.

4 Wright SE, Keeffe JE, Thies LS. Direct costs of blindness in Australia [in process citation]. Clin Exp Ophthalmol 2000;28:140-2.

5 Chiang YP, Bassi $\amalg$, Javitt JC. Federal budgetary costs of blindness. Milbank $Q$ 1992;70:319-40.

6 Wang JJ, Mitchell P, Smith W, et al. Impact of visual impairment on use of community support services by elderly persons: the Blue Mountains Eye Study. Invest Ophthalmol Vis Sci 1999;40:12-19.

7 Moisseiev J, Alhalel A, Masuri R, et al. The impact of the macular photocoagulation study results on the treatment of exudative age-related macular degeneration. Arch Ophthalmol 1995;113:185-9.

8 Treatment of age-related macular degeneration with photodynamic therapy (TAP) Study Group. Photodynamic therapy of subfoveal choroidal neovascularization in age-related macular degeneration with verteporfin: one-year results of 2 randomized clinical trials-TAP report. Arch Ophthalmol 1999;117:1329-45. 
9 Meads C, Salas C, Roberts T, et al. Clinical effectiveness and cost-utility of photodynamic therapy for wet age-related macular degeneration: a systematic review and economic evaluation. Health Technol Assess 2003; 7:1-108

10 Smith W, Assink J, Klein R, et al. Risk factors for age-related macular degeneration: Pooled findings from three continents. Ophthalmology 2001;108:697-704.

11 Mitchell P, Wang JJ, Smith W, et al. Smoking and the 5-year incidence of age-related maculopathy: the Blue Mountains Eye Study. Arch Ophthalmol 2002:120:1357-63

12 Mitchell P, Chapman S, Smith W. "Smoking is a major cause of blindness." A new cigarette packet warning? Med J Aust 1999;171:173-4.

13 White V, Hill D, Siahpush M, et al. How has the prevalence of cigarette smoking changed among Australian adults? Trends in smoking prevalence between 1980 and 2001. Tobacco Control 2003;12(Suppl 2):1167-74.

14 MMWR. Prevalence of current cigarette smoking among adults and changes in prevalence of current and some day smoking-United States, 1996-2001. Morb Mortal Wkly Rep 2003:52:303-7.

15 Marques-Vidal P, Ruidavets JB, Cambou JP, et al. Changes and determinants in cigarette smoking prevalence in southwestern France, 1985-1997. Eur J Public Health 2003;13:168-70.

16 AREDS report no 8. A randomized, placebo-controlled, clinical trial of highdose supplementation with vitamins $C$ and $E$, beta carotene, and zinc for agerelated macular degeneration and vision loss. Arch Ophthalmol 2001; 119:1417-36.

17 Barratt A, Irwig L, Glasziou P, et al. Users' guides to the medical literature: $\mathrm{XVII}$. How to use guidelines and recommendations about screening. EvidenceBased Medicine Working Group. JAMA 1999;281:2029-34.

18 Foran S, Wang JJ, Mitchell P. Causes of incident visual impairment: the Blue Mountains Eye Study. Arch Ophthalmol 2002;120:613-19.

19 McLennan W. Australian statistician. Population projections: 1997 to 2051 1-130. Canberra: Australian Bureau of Statistics, 1998.

20 Australian Bureau of Statistics. Deaths Australia 1996. ABS Catalogue 3302.0, 62-63. Canberra: ABS, 1997.
21 Sharma S, Brown GC, Brown MM, et al. Converting visual acuity to utilities. Can J Ophthalmol 2000;35:267-72.

22 Drummond MF, O'Brien B, Stoddart GL, et al. Methods for the economic evaluation of health care programmes, 2nd ed. New York: Oxford University Press, 1997.

23 Weinstein MC, O'Brien B, Hornberger J, et al. Principles of good practice for decision analytic modeling in health-care evaluation: report of the ISPOR Task Force on Good Research Practices - modeling studies. Value Health 2003;6:9-17.

24 Mitchell P, Smith W, Attebo K, et al. Prevalence of age-related maculopathy in Australia. The Blue Mountains Eye Study. Ophthalmology 1995; 102:1450-60.

25 Brown GC, Sharma S, Brown MM, et al. Utility values and age-related macular degeneration. Arch Ophthalmol 2000;1 18:47-51.

26 Brown MM, Brown GC, Sharma S, et al. Quality of life with visual acuity loss from diabetic retinopathy and age-related macular degeneration. Arch Ophthalmol 2002;120:481-4.

27 Brown MM, Brown GC, Sharma S, et al. Health care economic analyses and value-based medicine. Surv Ophthalmol 2003;48:204-23.

28 Sharma S, Oliver-Fernandez A, Bakal J, et al. Utilities associated with diabetic retinopathy: results from a Canadian sample. $\mathrm{Br} J$ Ophthalmol 2003;87:259-61.

29 Brown MM, Brown GC, Sharma S, et al. Utility values associated with blindness in an adult population. Br J Ophthalmol 2001;85:327-31.

30 Brown GC, Brown MM, Sharma S, et al. The reproducibility of ophthalmic utility values. Trans Am Ophthalmol Soc 2001;99:199-203.

31 Younis N, Broadbent DM, Vora JP, et al. Incidence of sight-threatening retinopathy in patients with type 2 diabetes in the Liverpool Diabetic Eye Study: a cohort study. Lancet 2003;361:195-200.

32 Laupacis A, Feeny D, Detsky AS, et al. How attractive does a new technology have to be to warrant adoption and utilization? Tentative guidelines for using clinical and economic evaluations. CMAJ 1992;146:473-81. 